\title{
Extracting group decision making experts' preferences from debate transcriptions using sentiment analysis
}

\author{
J. A. Morente-Molinera ${ }^{a}$ and F. J. Cabrerizo ${ }^{a}$ and I. J. Pérez ${ }^{b}$ and S. Alonso ${ }^{a}$ and E. Herrera-Viedma $^{a}$ \\ ${ }^{a}$ Andalusian Research Institute in Data Science and Computational Intelligence, \\ University of Granada, Spain, \\ jamoren@decsai.ugr.es, cabrerizo@decsai.ugr.es, zerjioi@ugr.es, viedma@decsai.ugr.es. \\ ${ }^{b}$ Department of Computer Sciences and Engineering, \\ University of Cádiz, Cádiz, Spain, ignaciojavier.perez@uca.es.
}

\begin{abstract}
Traditional group decision making methods work under the assumption that experts must provide their preferences to a computational system after carrying out a thorough debate. Nevertheless, this is not natural since the mean where experts express their ideas and preferences is inside the debate. In this paper, a novel method that is capable of extracting experts' preferences directly from the debate transcripts is presented. Thanks to this, experts do not have to go through a stressful process in order to provide the same information that they have already provide in the discussion part.
\end{abstract}

Keywords: Sentiment analysis, group decision making, linguistic modelling.

\section{Introduction}

Group decision making is a field that is quite present in the recent literature $[1,15,20]$. In a group decision making process, a set of experts need to rank a set of alternatives according to their adequateness to solve a certain problem. For this purpose, they provide preferences to the system after carrying out a thorough debate. The debate that the experts carry out is quite important since it gives the possibility of sharing different points of view and allow the experts to freely expose their opinions. Also, it helps them to reach an agreement before making a final decision $[11,24]$.

After carrying out the debate, experts provide their preferences to the system in order to know the final ranking of alternatives. For this purpose, they use means that the computer can interpret. These means are usually quite stiff and do not allow experts to clearly express themselves. Moreover, experts are being asked to provide information that they have already shared using their own means on the discussion process.

Taking into account how important and critical the discussion is for group decision making processes, it should be noticed that the computational system that implements the group decision making process does not usually interact with it. This is mainly by the following reasons:

- Discussions are not structured: The way that humans express themselves is full of inconsistencies and lack of a specific structure that a computational system can directly interpret. Therefore, there is a need of methods that aid the computational systems to understand the structures that the human language uses.

- Human language is difficult to interpret: Computers can only work with numbers. They cannot interpret words unless a specific framework is defined. This is exactly what linguistic modelling do [10]. By the use of fuzzy sets framework, linguistic modelling allows a computer to understand what a certain set of words mean. Nevertheless, although these frameworks are very useful, it is not possible for linguistic modelling to allow a computer to fully understand human language. It only allows the comprehension of a limited set of expressions.

In this paper, a novel method that allows a computational system to interpret texts from a group decision making debate in order to extract experts' preferences is presented. In order to achieve this goal, sentiment analysis procedures [13, 18] are used. Thanks to them, it is possible to analyze a text written in the human language and get an idea about how the person who wrote it was feeling. In group decision making processes, this fact becomes quite useful since it allows the system to understand how an 
expert was feeling when sharing a certain idea. With that information, the system can transform a debate transcription text into a preference value. Since this process is carried out automatically, there is no need for expert intervention and, consequently, they do not have to go under a tiresome process whose only purpose is to provide redundant information.

The paper is set as follows. In section 2, basis needed to understand the presented method are exposed. In section 3 , the developed method is presented. In section 4, an application example is shown in order to clarify how the presented method works. Finally, some conclusions are pointed out.

\section{Preliminaries}

\subsection{Group Decision Making}

Group decision making methods are quite popular in the recent literature $[1,2,11,20,24]$. Traditionally, group decision making methods were carried out by a set of experts that reunite in the same room in order to debate and sort all the possible alternatives. Nowadays, with the appearance of Web 2.0 technologies, group decision making methods need to adapt themselves in order to allow the experts to make decisions online. Web 2.0 allows experts to communicate and share their opinions using social networks. Thanks to them, the discussion can be performed in an organized way and all the experts' contributions are stored online. Thanks to this, any expert can read any opinion from any expert at any moment.

Formally, a Group Decision making process [6, 17] is the one where a set of experts $E=\left\{e_{1}, \ldots, e_{n}\right\}$ need to rank a set of alternatives $X=\left\{x_{1}, \ldots, x_{m}\right\}$ by using a set of preferences values, $P=\left\{p_{1}, \ldots, p_{n}\right\}$, that they provide to the system. These values contain the opinion of the experts about how the alternatives should be sorted.

In order to make a decision, the next steps are usually followed $[6,9]$ :

- Providing preferences: Experts provide their preferences to the system. Experts carry out a pairwise comparison of the alternatives [5, 14, 17]. and provide information about how much they prefer one alternative over another. Thanks to this, they do not have to focus on establishing a ranking of the whole set of alternatives at the same time.
- Calculating the collective preference matrix: Preferences provided by the experts are aggregated into a single collective matrix. This matrix contains information about all the preferences that have been provided by all the experts. In order to carry out the required computations, Ordered Weighting Averaging (OWA) operators [12] can be used. The resulting value is usually a matrix such that the position $p_{i j}$ contains the overall aggregation about how alternative $x_{i}$ is preferred over $x_{j}$.

- Measuring consensus: Consensus measures $[4,8]$ can be applied in order to determine if the experts have reached an agreement or if more debate is required. When the consensus is high, it means that the experts have reached an agreement and, therefore, they agree on a specific alternatives' ranking. On the contrary, if the consensus is low, then experts have different opinions and they should carry out more debate in order to bring opinions closer. It should be noticed that a decision that is being discussed and make all experts feel comfortable with it is better than a rush decision only supported by a simple majority. In the case that the experts cannot reach a high consensus value, it is always possible to set a maximum number of debate rounds. If that value is reached, then the decision process ends and the final ranking of alternatives is calculated.

- Creating the alternatives' ranking: Once that the consensus is high enough or if the maximum number of rounds have been reached, it is time to calculate the final ranking of alternatives. This step is performed by applying selection operators over the collective preference matrix that were calculated in the previous step. For instance, the guided dominance degree (GDD) operator [3] could be used.

The described process can be seen graphically on Figure 1.

In order to provide a preference representation mean that is easily understandable for experts, linguistic label sets are usually used in order to represent the information $[21,22,23]$. Thanks to them, the information is presented to the experts using words instead of numbers. Although linguistic modelling is more comfortable for the experts, it still has limitations. For instance, experts cannot express themselves using free text, they must follow the rules established by the linguistic label set used. That is, only words belonging to that sets are understandable for the computational system. Also, in a linguistic label set, the granularity is fixed. Therefore, the 


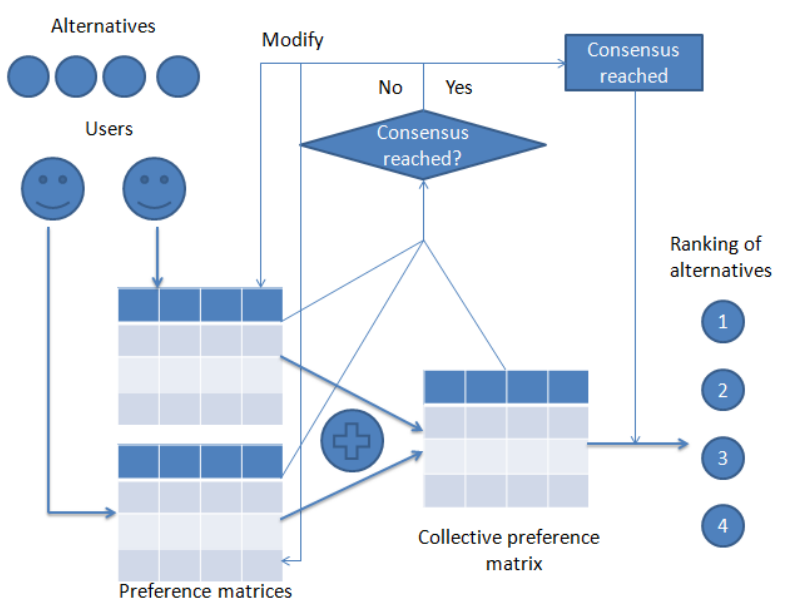

Figure 1: Group decision making scheme.

number of values of the linguistic scale is static. This can be solved by using multi-granular fuzzy linguistic modelling methods. Thanks to them, experts can select the linguistic label set that they want to use to provide their preferences.

In the presented methodology, experts are allowed to express themselves as they prefer. Thanks to sentiment analysis procedures, it is possible for the computational system to extract the experts' preferences from the debate transcriptions. This way, they do not have to explicitly provide information to the system.

\subsection{Sentiment analysis}

Creating methods that allow computational systems to comprehend human language is still nowadays a quite challenging task. Human language is full of inconsistencies and rhetoric that is difficult to understand and interpret by a computational system. In order to solve this issue, sentiment analysis procedures are designed. One of the most used sentiment analysis approaches is the lexicon-based one. This approach tries to understand what humans are experiencing when expressing themselves by analyzing the kind of words that they use. For instance, if a person uses words such as nice, beautiful or useful, then it is clear that the person has positive feelings about the topic that he/she is giving his/her opinion. On the contrary, if he/she employs negative words such as awful, nasty, terrible, then the person is not comfortable with the topic that he/she is talking about.

In order to identify sentiments over texts written in free language, the following procedure can be followed:

- Establishing the desired sentiment: First of all, it is necessary to identify which sentiments we are interested in. Depending on the sentiment, different words will be used to search for it.

- Generating a list of words: Lists of words that are typically used when experiencing each of the chosen sentiments is generated. The more words the list contains, the more precise the procedure will be.

- Analyzing free texts: Every word in the text that we are analyzing is searched on the generated lists. If the process finds out that several of that words are present in the list of words associated to a specific sentiment, then it means that the sentiment was present where the text were written.

- Presenting final results: Sentiments found are listed. It should be noticed that more than one sentiment can be associated to each analyzed text.

A scheme of the presented process is shown on Figure 2 .

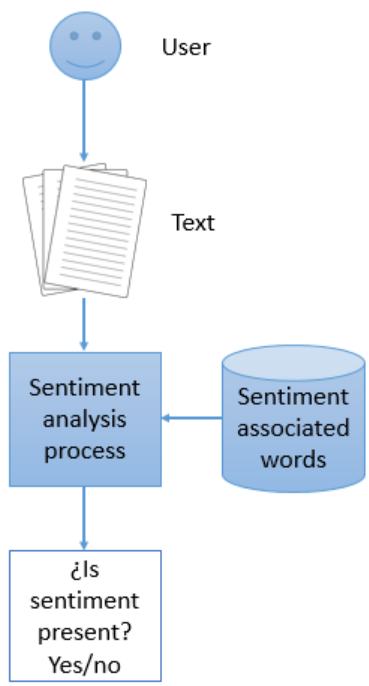

Figure 2: Sentiment analysis procedure scheme.

Sentiment analysis is a topic that is quite present in the recent literature. For instance, in, [7], a prototype tool that is capable of carrying out dynamic sentiment analysis of textual content from websites is presented. In [13], hierarchical fusion with context modelling is applied in order to solve multimodal sentiment analysis problems. Finally, in [18], a specific neural network structure is built in order to carry out sentiment analysis procedures. 


\section{$3 \quad$ Preferences extraction method}

The process followed to generate group decision making preferences from debate transcripts can be performed following the next two steps:

- Extracting debate transcriptions from the communication source: Transcriptions of the debate that the experts have carried out are obtained.

- Calculating experts' preferences: Once that the transcriptions texts have been obtained, it is possible to automatically transform that information into preferences that a computational system can interpret.

The presented steps are expose in detail in the following subsections.

\subsection{Extracting debate texts from the communication source}

The first thing to do in order to obtain experts' preferences from the debate transcriptions is to identify which are the sentences that experts have used in order to express opinions about the alternatives. In order to carry out this process, the following steps can be followed:

1. Defining a group decision making identifier: When the group decision making process is carried out over a specific mean, there it is clear that all the information available is related to it. Nevertheless, there are means, such as forums and social networks, where several discussions may be carried out in the same place and at the same time. If that is the case, it is necessary to define keywords that identify which contributions belong to the target group decision making process.

2. Identifying experts participating in the decision process: Once that the debate transcriptions that are related to the group decision making process that is being hold are identified, it is necessary to determine which expert has provided which contribution. This is usually a straightforward task since each contribution is typically assigned to a specific user. Specially in forums and social networks, there is no doubt which expert has provided each piece of information.

3. Obtaining the required debate texts: After the set of experts $E=\left\{e_{1}, \ldots, e_{n}\right\}$ and the sets of texts related to the group decision process, $\left\{A_{1}, \ldots, A_{n}\right\}$, have been identified, it is possible to initialize the process that extracts the preferences values from the texts.

\subsection{Calculating preferences}

Once that the information referring to the debate texts have been extracted, it is necessary to analyze it in order to estimate the preferences values that should be used in the group decision making process. In order to carry out this calculation, sentiment analysis procedures are applied. Since we are dealing with environments where experts can be in favor, against or have a neutral position about the alternatives, three different lists of words, $P L, N P L, S L$ are defined. $P L$ stores comparative expressions that are typically used when expressing than one thing is better than another. NPL stores comparative expressions indicating that one thing is worse than another. Finally, $S L$ stores expressions indicating that two elements are similar. Since it is expected that the experts express themselves in terms of comparative expressions, that is why these kinds of expressions are studied. It should be noticed that experts need to compare the alternatives in order to decide which ones are the best and generate the ranking.

Each of the used lists, $P L, N P L$ and $S L$ are built as tables where each row contains the following information:

- The comparative expression: The expression itself. If the words listed here appears in a text, it means that the expert has employed that expression to compare two elements.

- Comparison strength degree: All the comparative expressions do not have the same intensity. Some of them are more frequently used to express great differences among the alternatives and other ones to express little difference. According to the intensity, or comparison strength degree, a value is assigned to each expression. These values will be used for establishing the final preference value associated to the comparison of two specific alternatives.

Some examples of word lists entries can be observed on Table 1. In this case, the interval [1,5] has been used to assign the expression strength. 5 indicates the highest intensity while 1 indicates a value that is close to similarity between the alternatives. These lists should not be seen as static lists where the entries cannot be modified and are applicable to all the group decision making processes. On the contrary, all the information can be updated and the entries and strength values should be adapted to the topic that is being discussed. Finally, it is important to remark that $S L$ word list does not need strength values since all its expressions indicate 
Table 1: Example of comparative word list entries.

\begin{tabular}{|c|c|}
\hline Comparative & Value \\
\hline better & 4 \\
much better & 5 \\
cheaper & 2 \\
fairer & 2 \\
much fairer & 4 \\
\hline
\end{tabular}

neutrality between the alternatives. Therefore, a value of 0 is assigned.

Using word lists to identify sentiments is not the only way of achieving the pursued goal. Nevertheless, it is one of the fastest and most efficient way. Since experts want to obtain results without having to wait for too long, these are two characteristics that should be taken into account when choosing a specific sentiment analysis procedure. For testing purposes three specific lists have been generated. $P L$ has 304 entries, $N L$ has 308 and, finally, $S L$ has 116 expressions on it.

The process that the proposed approach follows to extract the preferences from the texts is specified below:

- Establishing keywords for the alternatives: First of all, it is necessary to identify which texts are related to which alternatives. For this purpose, a set of keywords, $K X=\left\{k x_{1}, \ldots, k x_{m}\right\}$, is defined for each alternative. Each $k x_{i}$ is formed by a set of words that clearly identify the alternative $x_{i}$ or that are used where the alternative is being mentioned.

- Identifying the comparative sentences that are related to the alternatives: The debate transcripts that have been selected in the previous subsection are analyzed in order to identify which sentences have some of the words that have been specified in the set $K X$. For every sentence and alternative, if, at least, one of the words is present on the sentence, then it is referring to the alternative referred by $k x_{i}$. As a result of this identification process, each expert $e_{i}$ has three sets of texts associated. First, $s p l_{i}$, which contains comparative expressions related to every two alternatives and belongs to $P L$. Second, snpl $l_{i}$, a set of sentences which contain expressions belonging to $N P L$. Finally, set $s s l_{i}$ stores the expressions which are located in $S L$. Each sentence must contain information about two alternatives and one comparative expression in order to be valid. The rest of the sentences are discarded from the process.

- Calculating a preference value in the interval $[-g, g]$ : Once that, for each expert and alter- native, the three sets, $s p l_{i}, s n p l_{i}$ and $s s l_{i}$, that indicate positive, negative and neutral sentences respectively are obtained, a preference matrix relation, $p_{i j}$, for each expert must be calculated. For this purpose, the strength values associated to the used expressions will be used. In the case that there are several expressions belonging to the same list valuing how alternative $x_{i}$ is preferred over $x_{j}$, then the most extreme value is chosen. For instance, if $x_{i}$ and $x_{j}$ has two associated expressions, one with a strength value of 3 and another one with a strength value of 4 , then the value 4 must be the chosen one. If no comparative expression is assigned for two specific alternatives, incomplete preference relations management methods can me employed to solve the issue [19]. From now on, it will be assumed that all the required information is present and, therefore, there is no need to estimate any preference value. Once that the strength value is assigned, the value is transformed depending on the list where the comparative expression belongs:

- If the comparative expression is from $P L$ : No change is applied to the value. For instance, if the strength value obtained is 3 then $p_{i j}=3$.

- If the comparative expression is from NPL: The obtained value is negated. For instance, if the strength value obtained is 2 , then $p_{i j}=-2$.

- If the comparative expression is from $S L$ : In this case, $p_{i j}=0$ no matter which comparative expression was found.

After performing the mentioned computations, a preference value belonging to the interval $[-\mathrm{g}, \mathrm{g}]$ is obtained. $g$ is the maximum value that can be assigned to the strength value $(g=5$ in our examples). With this new representation, a computational system can operate with the obtained results. Nevertheless, it would be useful to transform the $[-\mathrm{g}, \mathrm{g}]$ representation into one more typical for a linguistic label set.

- Representing the preference value into the desired linguistic label set: Although the actual representation is suitable for a computational system to interpret it, it can be desirable that the indexes do not contain negative values and that the linguistic label set has a different number of labels. In order to solve these issues, the following procedures can be applied:

- Removing negative values: In order to carry out an interval domain change in a way that the information represented in the interval $[-\mathrm{g}, \mathrm{g}]$ is transformed into information rep- 
resented in the interval $[0,2 \mathrm{~g}]$, the following formula can be applied:

$$
p_{i j}^{[0,(2 \cdot g)]}=p_{i j}^{[-g, g]}+g
$$

With the new transformation, $s_{0}$ indicates that alternative $x_{i}$ is totally not preferred to $x_{j}$. In a similar way, $s_{2 \cdot g / 2}$ indicates neutrality and $s_{2 \cdot g}$ exposes a total preference of $x_{i}$ over $x_{j}$.

- Changing the linguistic label set granularity: If the obtained granularity does not fit the experts' requirements, it is possible to change it using a multi-granular fuzzy linguistic modelling method [16]. Thanks to them, it is possible to express the information using the linguistic label set that experts prefer.

It should be noticed that the both procedures are optional. A graphical scheme of the presented process can be seen in Figure 3.

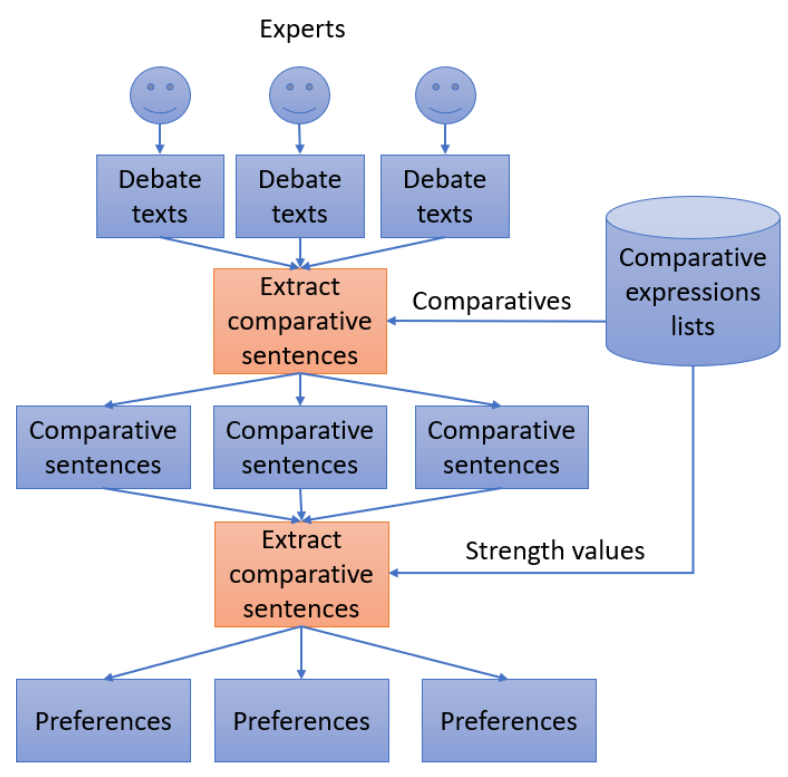

Figure 3: Preference obtainment scheme.

\section{Example}

In order to allow a better understanding of the presented method, an example is presented. Four experts $E=\left\{e_{1}, e_{2}, e_{3}, e_{4}\right\}$ need to decide where should some professors invest some recently acquired money. Four options are discussed, $X=\left\{x_{1}, x_{2}, x_{3}, x_{4}\right\}$. They are described in more detail in Table 3. First of all, the transcriptions of the debate are extracted.

The first step consists on establishing the sets $K X$. That is, the set of keywords used for identifying the alternatives on the extracted texts. In the third column of the Table 3, a set of keywords for the dealt alternatives is presented. The number of words that are suitable for the sets in $K X$ depends on the accuracy required to identify the alternatives on the texts.

Once that the texts have been extracted and the alternatives have been identified, the sentiment analysis procedure can begin. In Table 2, some examples of conversions from the free text to a linguistic label are presented. Strength values associated to the expressions are used in order to calculate the preference value that will be used in the group decision making process. Strength values of the comparative expressions that are used in Table 2 are shown in Table 4.

Expression 1 is used to calculate the linguistic labels. It should be noticed that the linguistic label set used for represent the preference values is: $S^{1} 0=\left\{s_{0}, \ldots, s_{1} 0\right\}$ and that strength values are represented using the interval $[0,5]$. Since values from expressions that belong to $N P$ are negated and values that belong to $S S$ have a strength value of 0 , then strength values shown in Table 4 belongs to the interval $[-5,5]$. The final preference value is shown in the second column of the Table.

\section{Conclusion}

In this paper, a novel way of obtaining preferences for a group decision making process is described. In traditional group decision making methods, it is quite typical that the experts have to debate and provide, at the same time, the discussion results to the system. This is redundant since in the discussion all the information and ideas have already been shared. Also, experts need to go under a process that is not comfortable for them. Thanks to the presented methodology, it is possible for the experts to focus on the discussion since the computational system can understand what they are trying to express thanks to sentiment analysis procedures.

For future work, the proposed theoretical framework will be tested in real scenarios.

\section{Acknowledgement}

The authors would like to thank FEDER financial support from the Project TIN2016-75850-P. 
Table 2: Sentences examples and their associated preference value.

\begin{tabular}{|l|c|c|}
\hline Sentences & Strn. value & Pref. value \\
\hline We need computers more than a new printer. & 4 & $p_{12}=s_{9}$ \\
\hline $\begin{array}{l}\text { Having a new office is a better option than going } \\
\text { to a conference. }\end{array}$ & 3 & $p_{34}=s_{8}$ \\
\hline $\begin{array}{l}\text { Going to a conference is preferable than to spend } \\
\text { it in having a new printer. }\end{array}$ & 4 & $p_{42}=s_{9}$ \\
\hline $\begin{array}{l}\text { It is better to buy a new printer than to invest in } \\
\text { having a new office. }\end{array}$ & 0 & $p_{23}=s_{9}$ \\
\hline $\begin{array}{l}\text { It would be similar to me to acquire a new office or } \\
\text { a new printer }\end{array}$ & -3 & $p_{32}=s_{5}$ \\
\hline $\begin{array}{l}\text { It would be worse for me to spend the money in a } \\
\text { conference than to buy a new printer }\end{array}$ & & \\
\hline
\end{tabular}

Table 3: Alternatives descriptions.

\begin{tabular}{|c|c|c|}
\hline $\mathbf{X}$ & Description & Keywords \\
\hline$x_{1}$ & More computers & computer, pc, laptops \\
$x_{2}$ & New printer & printer, printing \\
$x_{3}$ & Acquire new offices & room, office \\
$x_{4}$ & Going to a conference & conference, special session \\
\hline
\end{tabular}

Table 4: Comparatives and associated preference values.

\begin{tabular}{|c|c|}
\hline Comparative & Strn. value \\
\hline need more than & 4 \\
better option & 3 \\
preferable & 4 \\
better & 4 \\
similar & - \\
worse & 3 \\
\hline
\end{tabular}

\section{References}

[1] N. Banaeian, H. Mobli, B. Fahimnia, I. E. Nielsen, M. Omid, Green supplier selection using fuzzy group decision making methods: A case study from the agri-food industry, Computers \& Operations Research 89 (2018) 337-347.

[2] G. Baudry, C. Macharis, T. Vallee, Range-based multi-actor multi-criteria analysis: A combined method of multi-actor multi-criteria analysis and monte carlo simulation to support participatory decision making under uncertainty, European Journal of Operational Research 264 (1) (2018) $257-269$.

[3] F. J. Cabrerizo, R. Al-Hmouz, A. Morfeq, A. S. Balamash, M. Martínez, E. Herrera-Viedma, Soft consensus measures in group decision making using unbalanced fuzzy linguistic information, Soft Computing 21 (11) (2017) 3037-3050.
[4] F. J. Cabrerizo, F. Chiclana, R. Al-Hmouz, A. Morfeq, A. S. Balamash, E. Herrera-Viedma, Fuzzy decision making and consensus: challenges, Journal of Intelligent \& Fuzzy Systems 29 (3) (2015) 1109-1118.

[5] F. J. Cabrerizo, E. Herrera-Viedma, W. Pedrycz, A method based on PSO and granular computing of linguistic information to solve group decision making problems defined in heterogeneous contexts, European Journal of Operational Research 230 (2013) 624-633.

[6] N. Capuano, F. Chiclana, H. Fujita, E. HerreraViedma, V. Loia, Fuzzy group decision making with incomplete information guided by social influence, IEEE Transactions on Fuzzy Systems 26 (3) (2018) 1704-1718.

[7] I. M. de Diego, A. Fernández-Isabel, F. Ortega, J. M. Moguerza, A visual framework for dynamic emotional web analysis, Knowledge-Based Systems 145 (2018) 264-273.

[8] C. De Maio, G. Fenza, V. Loia, F. Orciuoli, Linguistic fuzzy consensus model for collaborative development of fuzzy cognitive maps: a case study in software development risks, Fuzzy Optimization and Decision Making 16 (4) (2017) 463479.

[9] Y. Dong, S. Zhao, H. Zhang, F. Chiclana, E. Herrera-Viedma, A self-management mechanism for non-cooperative behaviors in large-scale 
group consensus reaching processes, IEEE Transactions on Fuzzy Systems 26 (6) (2018) 32763288 .

[10] C.-C. Li, Y. Dong, F. Herrera, E. HerreraViedma, L. Martínez, Personalized individual semantics in computing with words for supporting linguistic group decision making. an application on consensus reaching, Information Fusion 33 (2017) 29-40.

[11] F. Liu, Y.-H. Wu, W. Pedrycz, A modified consensus model in group decision making with an allocation of information granularity, IEEE Transactions on Fuzzy Systems 26 (5) (2018) 3182 3187 .

[12] X. Liu, B. Han, H. Chen, L. Zhou, The probabilistic ordered weighted continuous owa operator and its application in group decision making, International Journal of Machine Learning and Cybernetics (2017) 1-11.

[13] N. Majumder, D. Hazarika, A. Gelbukh, E. Cambria, S. Poria, Multimodal sentiment analysis using hierarchical fusion with context modeling, Knowledge-Based Systems 161 (2018) 124-133.

[14] J. Morente-Molinera, G. Kou, R. GonzálezCrespo, J. Corchado, E. Herrera-Viedma, Solving multi-criteria group decision making problems under environments with a high number of alternatives using fuzzy ontologies and multi-granular linguistic modelling methods, Knowledge-Based Systems 137 (2017) 54-64.

[15] J. A. Morente-Molinera, G. Kou, Y. Peng, C. Torres-Albero, E. Herrera-Viedma, Analysing discussions in social networks using group decision making methods and sentiment analysis, Information Sciences 447 (2018) 157-168.

[16] J. A. Morente-Molinera, I. J. Pérez, M. R. Ureña, E. Herrera-Viedma, On multi-granular fuzzy linguistic modeling in group decision making problems: a systematic review and future trends, Knowledge-Based Systems 74 (2015) 49-60.

[17] J. A. Morente-Molinera, R. Wikström, E. Herrera-Viedma, C. Carlsson, A linguistic mobile decision support system based on fuzzy ontology to facilitate knowledge mobilization, Decision Support Systems 81 (2016) 66-75.

[18] K. Shuang, Z. Zhang, H. Guo, J. Loo, A sentiment information collector-extractor architecture based neural network for sentiment analysis, Information Sciences 467 (2018) 549-558.
[19] R. Ureña, F. Chiclana, J. A. Morente-Molinera, E. Herrera-Viedma, Managing incomplete preference relations in decision making: A review and future trends, Information Sciences 302 (2015) $14-32$.

[20] J. Wu, L. Dai, F. Chiclana, H. Fujita, E. HerreraViedma, A minimum adjustment cost feedback mechanism based consensus model for group decision making under social network with distributed linguistic trust, Information Fusion 41 (2018) 232-242.

[21] L. A. Zadeh, The concept of a linguistic variable and its application to approximate reasoning-I, Information sciences 8 (3) (1975) 199-249.

[22] L. A. Zadeh, The concept of a linguistic variable and its application to approximate reasoning-II, Information sciences 8 (4) (1975) 301-357.

[23] L. A. Zadeh, The concept of a linguistic variable and its application to approximate reasoning-III, Information sciences 9 (1) (1975) 43-80.

[24] H. Zhang, Y. Dong, E. Herrera-Viedma, Consensus building for the heterogeneous large-scale gdm with the individual concerns and satisfactions, IEEE Transactions on Fuzzy Systems 26 (2) (2018) 884-898. 